\title{
5 Rational Exploitation of the Core by the Periphery? On the Collective (In)efficiency of Endogenous Enforcement of Universal Conditional Cooperation in a Core-Periphery Network
}

\begin{abstract}
Raub and Weesie (1990) proposed a game theoretical model addressing effects of network embeddedness on conditional cooperation between two actors. This work showed that network embeddedness can facilitate conditional cooperation by reducing uncertainty, in line with a number of follow-up contributions and consistently with results from other modelling approaches. This research focused mainly on interactions between two parties embedded in a network. In the present paper, I extend a closely related model towards $N$-person collective good problems, combining conditional cooperation based on direct monitoring via network ties and observation of group output in an uncertain environment. The focus is on a maximally simple yet empirically relevant case, a core-periphery network in which only core-members can directly observe each other's contributions to a collective effort, whereas peripheral members only observe a noisy signal indicating aggregated contributions. I propose the possibility of a 'rational exploitation of the core by the periphery'. Strategy-profiles in which free-riding of peripheral members is tolerated while core-members cooperate conditionally, are not only individually rational but also payoff-superior to profiles with universal conditional cooperation if uncertainty is sufficiently high and the number of peripheral members is sufficiently low.
\end{abstract}

\subsection{Introduction}

The question under which conditions cooperation can be achieved in the production of collective goods is still prominently on the agenda of social scientists more than five decades after Olson's (1965) influential analysis of the problem of collective action. Especially for "large number dilemmas" (Raub 1988), Olson and many

\footnotetext{
Notes: I am greatly indebted to Werner Raub for the inspiration and guidance over the years that has formed the basis for this work and for so much more. I also thank the editors for a thorough review and constructive comments on an earlier version of the manuscript.
}

Andreas Flache, University of Groningen, Department of Sociology / ICS 
other authors (for example Hardin 1968) were pessimistic about the possibility of an endogenous solution without hierarchical enforcement. However, game theoretical research has shown that reciprocity in the form of conditional cooperation can be an individually rational endogenous solution of collective action problems (Friedman 1971, 1986; Raub and Voss 1986; Raub 1988; Taylor 1976, 1987) in a collective action situation that constitutes an infinitely or indefinitely repeated game.

Conditional cooperation in collective good production makes an actor's contribution to the common effort contingent upon others' contributions in the past, similar to the well-known strategy of "Tit-for-Tat" (Axelrod 1984) in 2-person social dilemma games. An important problem that can limit the effectiveness of conditional cooperation is that actors may not be able to observe precisely and reliably others' past contribution (Bendor and Mookherjee 1987; Green and Porter 1984). For example, when a project team member in an organization fails to show up for a project meeting, this may be intended free-riding and should thus trigger retaliation from other team members to credibly deter future free-riding according to the logic of conditional cooperation. But possibly the absent team member was delayed by an incident he cannot be held accountable for, like unexpected sickness of a child. What makes things worse, it is often hard to verify for other actors involved what the true reasons were if someone failed to contribute, or who in a team was responsible when the team's results failed to meet expectations. The dilemma here is that sanctions are needed in such a situation to deter free-riding behavior. But such sanctions also bear the danger of putting successful cooperation under pressure, for example when the target of a sanction feels treated unfairly and responds with a counter-sanction (Nikiforakis 2008; Nikiforakis and Engelmann 2011) potentially evoking cycles of mutual recrimination (Bendor, Kramer and Stout 1991; Kollock 1993; Wu and Axelrod 1995) disrupting ongoing cooperation more than necessary.

Network embeddedness (Granovetter 1985) can mitigate the difficulties that arise from information problems in ongoing collective action. Network ties connecting some of the participants of the collective action more closely than others, such as friendship relations between some team members, or close physical proximity of workplaces or homes, give some the opportunity to observe more reliably whether their network contacts contributed to a joint effort or for which reasons they failed to do so. Moreover, network ties allow to communicate this information to further participants of the collective action. As a result, a network of interpersonal ties may stabilize conditional cooperation in a situation where contributions are hard to observe without direct network connections, because either through direct observation or communication via network relations participants know better why failures to contribute occurred and sanctions can thus be more effectively directed at free riders.

Raub and Weesie (1990) developed a game-theoretical model formalizing this argument and showed how and under which conditions social networks facilitate trust in cooperation problems faced in two-party relations (see also Raub and Weesie, 
2000), as they occur for example in business relations or between partners in a household. A range of follow-up studies extended this analysis and tested the argument empirically (Batenburg, Raub and Snijders 2003; Buskens 2002; Buskens and Raub 2002; Raub 2017; Raub and Buskens 2008; Rooks, Raub, and Tazelaar 2006). This work contributed to a wider literature of models that link network ties to cooperation. Most of that literature focuses on mechanisms other than conditional cooperation. Some examples are imitation (Gould 1993), threshold dynamics (Chwe 1999; Macy 1991), mobilization via network ties (Marwell and Oliver 1993), sanctions imposed via social ties (Coleman 1990; Flache 1996; Flache, Macy, and Raub 2000), or information on others' preferences acquired via network relations (Dijkstra and van Assen 2013). Some authors also modelled conditional cooperation in repeated collective good games (Bednar 2006; Bendor and Mookherjee 1990; Flache 2002; Flache et al. 2000; Spagnolo 1999; Wolitzky 2013). Especially Fatas and co-authors (for example Fatas, Meléndez-Jiménez, and Solaz 2010; Fatas et al. 2015) developed a line of papers combining theoretical modelling and experimental tests of effects of networks on conditional cooperation in collective good settings.

Despite some exceptions, the problem of cooperation in collective goods has received relatively little attention in the research that links network embeddedness to the feasibility of conditional cooperation under uncertainty, compared to the problem of cooperation in two-party relations. One possible reason is that models of conditional cooperation in collective action typically assume that in addition to networks there is another source of information actors can rely upon to condition their own contribution behavior on others' cooperation. This is the level of the good provided, indicating how many group members made a contribution to bring it about. A number of theoretical studies (Friedman 1971; Raub 1988; Raub and Voss 1986; Taylor 1987) highlighted that this information is under certain conditions sufficient to render it an individually rational endogenous outcome of the game if everyone cooperates conditionally upon sufficient group-output. But imperfect information provides an important problem also for this endogenous solution. Observed levels of provision of a collective good are rarely a perfect or reliable indicator of how much effort group members really have invested to bring the good about. Bendor and Mookherjee (1987; see also Bendor, Kramer and Stout 1991; Kollock 1993) prominently showed how this can make conditional cooperation highly inefficient. If the condition for cooperation is sufficient group-output, this implies that under uncertainty sometimes the condition may not be met for reasons which are unrelated to deliberate free-riding by some group members - like the unintended failure of a team member to turn up for a project meeting. Rational actors face the dilemma that therefore sanctioning strategies must be lenient to some extent to avoid too much 'unnecessary' mutual punishment, but that too much lenience invites deliberate defection because deviants can hope to 'get away' with occasional free-riding. In other words, the social costs of enforcing cooperation by sufficiently severe sanction threats may become prohibitively high if there is too much uncertainty about 
the link between efforts actors make to contribute to a collective good and the observable results in terms of the level of provision.

Work on the effect of uncertainty for conditional cooperation suggests that monitoring in social networks can be an important factor that stabilizes conditional cooperation in collective action. Yet, hitherto only few studies address the role of monitoring via network ties in a setting where actors simultaneously observe a group-output that is an unreliably indicator of actual contributions. In this paper, I try to take a step in that direction by proposing a game-theoretical model of collective action under uncertainty, aiming to integrate effects of monitoring via social network ties albeit with a maximally simple network structure.

In modelling the network structure in a maximally simple way, I want to explore a structural problem that occurs in many situations where collective action is needed. Often, networks are heterogeneous in the extent to which their members are connected among each other.

One particular case of heterogeneity are core-periphery structures, in which some members of an interest group form a densely connected core and others are in peripheral positions with only sparse links to members in the core. Consider as an extremely simple case the situation in which core members can monitor each other's contributions to the collective effort very closely and accurately, while the effects of the contributions of peripheral members are only visible via their impact on the group-output, while their real efforts to contribute to the collective good are private information. Empirical settings for which this could be seen as an idealtypical model of a collective good situation might be (a) a company that works with staff located in headquarters and local representatives dispersed across different regions or countries, (b) a semi-virtual organization, working with a local core team of members physically located in an office, and a number of workers who are only connected online with each other and the core-members (Flache 2004), or (c) a local renewable energy initiative largely driven by a core-team of densely connected 'front-runners' but in need of contributions from a larger number of members of the community who are much less connected with the front-runners than they are connected with each other (Goedkoop, Flache, and Dijkstra 2017).

Core-periphery structures can impose a dilemma for endogenous conditional cooperation. Under uncertainty, sanctioning regimes that provide sufficient incentives for all members to cooperate in equilibrium cannot avoid that some punishment must be imposed even when only 'erroneous' defections occur, because with imperfectly observed input of the peripheral members their free riding can otherwise not be credibly deterred. In this situation the closer embeddedness of the core-members can make an alternative regime more attractive, a sanctioning regime in which coremembers ignore the unreliable and noisy information about collective output, but condition their behavior instead on the reliably observed inputs of only the other core-members. Obviously, the problem with that alternative solution is that peripheral members can no longer be credibly deterred from free riding. They are effectively 
allowed to take a free-ride, an outcome that I call here exploitation of the core by the periphery, mirroring Olson's (1965) 'exploitation of the big by the small'. What makes this possibility nevertheless worth to investigate is that it may under certain conditions be collectively more efficient than a conditional cooperation regime in which sanctions can be triggered by everyone's failure to contribute. Intuitively, the reason is that credible enforcement of the cooperation of those group members whose actions are difficult to observe is not possible without risking a considerable amount of sanctions that are triggered by unintended failures to generate effective contributions. Imposing such a sanction is damaging and potentially disruptive in itself for the collective effort. Tolerating some free riding from peripheral members avoids these costs, potentially rendering such tolerance a collectively more desirable solution. As an example, consider the renewable energy initiative discussed above. If every time when not enough community members show up for an information meeting about the initiative, the core-team of front-runners would stop its activities for some time to sanction 'freeriders' in the community, the initiative may suffer more damage from those sanctions than was caused by the lack of contribution of peripheral community members in the first place. Accepting relatively low turn-out at information meetings may thus be a collectively more efficient strategy of the front-runners than trying to enforce contributions from all community members all the time.

In what follows I investigate the conditions under which the outcome in which peripheral members free ride and core-members contribute can be both individually rational as well as socially more efficient than universal conditional cooperation. The model I use for this will be described in Section 5.2, results for specific scenarios are presented in Section 5.3 and the paper closes with a discussion of possible implications and limitations in Section 5.4.

\subsection{Model}

In section 5.2.1. the repeated game is presented, section 5.2.2. describes the approach for analysis of the conditions for individual rationality and social efficiency of the alternative outcomes of universal conditional cooperation on the one hand, and "core-only" conditional cooperation on the other hand.

\subsubsection{The repeated game}

Group interaction is modelled as a repeated $N$-person game that is equivalent to the "work game" in Flache (2002), except for the assumption that monitoring of contribution behavior is possible via direct network-ties. The constituent-game strategy of player $i$ in iteration $t$ of the repeated game is represented by the decision $w_{i t}$ 
whether to "work" or "shirk", where $w_{i t}=0$ for defectors and $w_{i t}=1$ for contributors. In the stage game, actors take decisions simultaneously and independently.

Following Bendor and Mookherjee (1987) uncertainty is modelled with a commonly known universal probability $\varepsilon$ that due to some mishap an individual's contribution fails to be effective $(0 \leq \varepsilon \leq 1)$. It is assumed that all players know after every iteration $t$ the group-output in terms of the number of effective contributions group members made in $t$, but players are not necessarily aware of the actual input $w_{j t}$ of other individual members.

Modelling the core-periphery structure, I assume a maximally simple social network $S$. The group consists of $N_{c}$ core-members and $N_{i}$ isolates $\left(N=N_{c}+N_{i}\right)$. Coremembers are connected to all other core-members in the network, whereas isolates are not connected to anyone else. If there is a connection between individuals $i$ and $j$ $\left(s_{i j}=1\right), i$ is at time $t$, before taking her own decision, fully and perfectly informed on the actual contribution decision $w_{j t}$ her network contact $j$ made in all previous iterations $t^{\prime}<t$. This is a rather extreme simplification, but it greatly facilitates model analysis, while it still captures the substantive assumption that monitoring contributions via network relations is more reliable than between unconnected actors.

The expected payoff of actor $i$ in iteration $t$ of the game, $u_{i t}$, results from both expected benefits from output and expected costs of $i$ 's own contribution-effort. Output is simply modelled as a linear function of the sum of individual outputs. Notice that output may be lower than the number of actual contributions made, due to uncertainty. The expected output generated by an individual contribution is $(1-\varepsilon) w_{i t}$. The amount of the collective good produced is shared equally among all group members, whereas costs of making a contribution are private. Equation (5.1) formalizes the expected payoff an individual derives from the outcome of the constituent game in iteration $t$ :

$$
u_{i t}=\sum_{j=1}^{N}\left(\frac{\alpha}{N}(1-\varepsilon) w_{j t}-c w_{i t}\right) .
$$

The parameter $\alpha$ scales the benefit a group member receives from consuming a unit of the collective good. The costs of investing a unit of effort into its production are indicated by the parameter $c$. Modelling a problematic collective action situation, the constituent game has a $N$-person Prisoner's dilemma structure given by $\alpha / N<c$ $<\alpha$.

To model conditional cooperation in the repeated game, I use the standard assumption of infinite repetition of the game with exponential discounting of future payoffs. The accumulated payoff $u_{i}$ of actor $i$ in the repeated game sums discounted payoffs over all iterations $t, u_{i t}$. Formally,

$$
u_{i}=\sum_{t=0}^{\infty} \tau^{t} u_{i t}, \quad 0<\tau<1 .
$$


where $\tau$ is the discount parameter, defining the value of players' interest in future payoffs. For simplicity, $\tau$ as well all the parameters $\alpha, \varepsilon$ and $c$ are assumed equal for all members of the group.

\subsubsection{Model analysis}

\subsubsection{Strategy types}

Main aim of the model analysis is comparison of the conditions for individual rationality and social efficiency of two types of strategy profiles for the repeated game, modelling two different types of reciprocity norms. In this game, the only 'weapon' group members have to impose a sanction on defectors is response with own defection. Conditional cooperation thus implies reciprocal behavior in the sense that all players cooperate as long as there has been sufficient cooperation by others in the past, but resort to defection otherwise. The first type of strategy profile analyzed here meets the additional requirement of symmetry. The stage-game behavior expected from players, the attached conditions for triggering a sanction and the severity of the sanction are universally shared in this profile. I call the corresponding strategy-type "universal conditional cooperation" in what follows. Especially, both core-members and isolates follow the same reciprocity norm under universal conditional cooperation. This can be seen as a property that ensures 'procedural fairness', a key ingredient for sustainable cooperation according to many authors. The second type of strategy profile relaxes the 'procedural fairness' requirement of symmetry for the sake of potential gains in social efficiency. This type differentiates between the norm imposed upon core-members and a maximally lenient norm imposed upon isolates, effectively allowing them to free-ride. I call this type "core-only" conditional cooperation. Both strategy-types as well as their analysis are discussed in more detail below.

\subsubsection{Individual rationality and social efficiency of universal conditional cooperation}

A key principle for the selection of strategy profiles as solutions of the game is individual rationality. Technically, this implies that the solution of the game is a subgame perfect Nash equilibrium (s.p.e.) (Selten 1965; see also Kreps 1990). Another key principle is social efficiency in terms of payoff dominance. Payoff dominance eliminates those s.p.e.'s from the set of possible solutions of a game to which all players would unanimously prefer other s.p.e.'s (for more details, see Harsanyi 1977:116-119). Among the set of individually rational and symmetric reciprocity strategy profiles, those will be selected as candidate-solution of the game that yield a higher payoff to all members of the group compared to other strategy-profiles in 
this set. Given symmetry, this means the s.p.e. will be selected that maximizes $u_{i}$ as defined by (5.2) for all players.

The analysis uses a generalized form of trigger strategies (Friedman 1971, 1986), following Bendor and Mookherjee (1987; see also Flache 2002). Trigger strategies under imperfect information generate cooperative behavior in a normal period of the game, but as soon as the corresponding group-output norm has been violated, the trigger strategy reverts to a punishment behavior for a subsequent sanctioning period. After the sanctioning period, cooperation is restored but only as long as there is sufficient group-output. Two parameters of a trigger strategy model the tradeoff between lenience and deterrence, the cutoff level $l$ and the sanction time $s$. In the strategy-profile $\sigma(s, l)$ all players cooperate in all rounds in a normal period, but they revert to a sanctioning period of exactly $s$ rounds, as soon as in the normal period the group-output falls below the cut-off level $l$. After the sanctioning period, a new normal period starts with an initial round of unconditional cooperation.

To guarantee individual rationality of adhering to $\sigma(s, l)$, the profile needs to ensure that optimal unilateral deviations from it do not pay. To also optimize efficiency, the strategy profile $\sigma\left(s^{*}, l^{*}\right)$ is sought that maximizes the related expected payoff $u_{i}\left(\sigma\left(s^{*}, l^{*}\right)\right)$, subject to the constraint that the corresponding trigger strategy constitutes a subgame perfect equilibrium. ${ }^{1}$

For calculation of expected payoffs of trigger-strategies and for evaluation of the constraint that unilateral deviations do not pay, I adapted an efficient numerical algorithm from Bendor and Mookherjee (1987) that solves the optimization problem for a given set of conditions. $\left(\alpha, c, N, N_{c}, \varepsilon, \tau\right)$. The algorithm will here only be sketched in broad strokes, more details can be found in Flache (2002). The analysis of the trigger strategy profile "task-cooperation" in Flache (2002:199) is equivalent to the analysis of $\sigma(s, l)$ here.

The algorithm finds the payoff-dominant s.p.e. in the set of $\sigma(s, l)$ profiles in two steps. In the first step, it is established for which cut-off levels $l$ ' individually rational profiles $\sigma\left(s, l^{\prime}\right)$ can exist. Such a profile exists if and only if the condition is satisfied that the trigger strategy $\sigma(\infty, l$ ') with eternal punishment and cut-off level $l$ ' is

1 Due to symmetry, trigger strategies always constitute a subgame perfect equilibrium, if and only if they satisfy the conditions for Nash-equilibrium. The proof is given by Friedman (1986). For the game at hand here a possible complication is that core-members have more information about possible deviations than isolates have. Off the equilibrium path, a core-member can observe deviations by other core-members even if those deviations do not lead to a sufficient drop in output to trigger a subsequent punishment phase under $\sigma\left(s^{*}, l^{*}\right)$. The core-member might thus have an incentive to deviate from the profile $\sigma\left(s^{*}, l^{*}\right)$ in the subgame that ensues, because the observed deviation changes the probability that there will be a punishment phase in the next round. To avoid such complications I assume that both group-output and individual inputs become known only after all stage-game decisions were taken and before the next round begins. This makes it public knowledge for all group-members whether in the subsequent round a punishment phase will start, aligning the conditions for deviation in the subgame with those for deviation in the overall game. 
individually rational. Intuitively, the reason is that eternal punishment maximizes the expected loss from the sanction that a deviant faces. If eternal damnation is not sufficient to deter deviation at l', no finite sanction time $s$ is. Theorem 1 in Flache (2002) gives the condition that follows, which in turn is efficiently tested with the numerical algorithm for all feasible cut-off levels $l^{\prime}, 1 \leq l ' \leq N$.

The second step of the numerical procedure is to calculate and compare the optimal expected payoffs from universal conditional cooperation that can be obtained for those cut-off levels $l^{\prime}$ that satisfy the condition that $\sigma\left(\infty, l^{\prime}\right)$ is s.p.e. The optimal profiles $\sigma\left(s^{\prime}, l^{\prime}\right)$ can be found by inspection of only one trigger strategy per cut-off level $l$, the one that minimizes sanction time $s$, subject to the constraint of individual rationality $u_{i}-u_{-i}$ under $l=l$ ', where $u_{i}$ and $u_{-i}$ denote the payoffs for universal cooperation and the optimal unilateral deviation from that profile for $i$, respectively. Shorter sanctioning periods ensure higher payoffs for all under universal conditional cooperation. But there is also a critical lowest sanction time $s^{*}$, below which the individual rationality constraint $u_{i} \geq u_{-i}$ can no longer be satisfied because sanctions become too lenient. Theorem 2 in Flache (2002) specifies how this sanctiontime $s^{*}$ is efficiently computed by the numerical algorithm.

Given a vector of parameters $\left(\alpha, c, N, N_{c}, \varepsilon, \tau\right)$ of the game, the algorithm finds the payoff-dominant s.p.e. $\sigma\left(s^{*}\left(l^{\prime}\right), l^{\prime}\right)$ for every cut-off level $l^{\prime}$ for which an individually rational profile $\sigma\left(s, l^{\prime}\right)$ exists. Among these payoff-dominant profiles $\sigma\left(s^{*}\left(l^{\prime}\right), l^{\prime}\right)$, the algorithm then selects the cut-off level $l^{*}$ that maximizes the related expected payoff. If no other s.p.e. exists for the given set of parameters, the unique symmetric solution of the game is universal and full defection.

\subsubsection{Individual rationality of core-only conditional cooperation}

The core-only strategy-profile $\sigma_{c o}$ implies unconditional contribution to the collective good in the first round of the game for core-members. Thereafter, coremembers contribute if and only if all other core-members have contributed in all previous rounds of the game. That is, as soon as a core-member detects defection by another core-member, all core-members revert to eternal defection. Core-members condition their behavior exclusively upon the observed actions of other core-members. In what follows, the analysis will be restricted to the most extreme form of a "core-only" strategy-profile, the form in which all isolates choose full defection. The conditions under which such a profile is individually rational for core-members can be seen as the most restrictive conditions under which individually rational profiles exist at all that demand less cooperation from isolates than from core-members. If core-members are willing to adopt this core-only strategy, strategy profiles with higher levels of cooperation by isolates would yield higher payoffs for all core members and thus also be individually rational for them. Alternatively, isolates could adopt a $\sigma(s, l)$ profile in which they would enforce some level of conditional cooperation from each other with the 
threat of sanctioning if group-output drops too low. Yet, at least as long as isolates are in the minority, this would impose only a relatively weak sanction because groupoutput drops only by $(1-\varepsilon) N_{i}$. in sanctioning periods. This suggests that conditions under which such a profile is individually rational also for isolates are rather restrictive and demand very long sanctioning periods, which diminishes possible efficiency gains compared to the extreme case of full defection by isolates.

The condition for individual rationality of cooperation among only the coremembers in the strategy-profile $\sigma_{c o}$ follows from Friedman's theorem $(1971 ; 1986$ : 88-89) for indefinitely repeated games with perfect monitoring of past behavior. $\sigma_{c o}$ constitutes a subgame perfect equilibrium, if and only if core-members are sufficiently interested in future payoffs. This the condition given by equation (5.3).

$$
\tau>\tau^{*}=\frac{\hat{T}_{c o}-\hat{R}_{c o}}{\hat{T}_{c o}-P} .
$$

The symbol $\hat{T}_{c o}$ denotes the expected stage-game payoff from unilateral deviation by a core-member, $\hat{T}_{c o}=(1-\varepsilon) \alpha\left(N_{c}-1\right) / N$. The symbol $\hat{R}_{c o}$ refers to the stage-game payoff for a core-member of universal cooperation by only core-members, $\hat{R}_{c o}=(1-\varepsilon) \alpha N_{c} / N-c . P$, finally, is the stage-game payoff of universal defection for a core-member, which is zero.

\subsubsection{Solution of the game}

The solution of the game that is selected for a given parameter-vector $\left(\alpha, c, N, N_{c}, \varepsilon, \tau\right)$ is full defection by all players if neither any $\sigma(s, l)$ nor $\sigma_{c o}$ constitute an s.p.e. If only $\sigma_{c o}$ constitutes an s.p.e. this is the solution, if only $\sigma(s, l)$ profiles are s.p.e. then the payoff-dominant solution $\sigma\left(s^{*}, l^{*}\right)$ is the solution. Finally, if both $\sigma(s, l)$ profiles and $\sigma_{c o}$ constitute s.p.e.'s, then the payoff dominant one among the set of all these s.p.e.'s is selected as solution. Notice that for $\sigma_{c o}$ this requires in particular that the accumulated payoff is higher both for core-members and for isolates compared to the payoff all members receive in $\sigma\left(s^{*}, l^{*}\right)$. Accumulated payoff as well as the incentive to deviate, $u_{i}-u_{-i}$ for $\sigma_{c o}$ can be obtained from $u_{i t}=\hat{R}_{c o}, u_{-i t}=\hat{T}_{c o}$ and equation (5.2). Accumulated payoff $u_{i}$ and incentive to deviate, $u_{i}-u_{-i}$ for $\sigma(s, l)$ profiles are computed as given in Flache (2002).

\subsection{Results}

First, it will be explored for an illustrative scenario how changes in the 'shadow of the future' $\tau$, the number of isolates $N_{i}$, and the degree of uncertainty $\varepsilon$ affect the conditions for individual rationality and efficiency of a specific trigger-profile $\sigma\left(s^{*}, l^{*}\right)$ 
compared to $\sigma_{c o}$. Second, comparative statics will be reported about the effects of these variables on the distribution of optimal solutions across the entire range of possible values of $\tau$.

\subsubsection{Illustrative scenario}

The scenario inspected here represents a relatively small work-team with a minority of isolates, facing a moderately severe cooperation problem under considerable uncertainty, formalized as $N=10 ; \varepsilon=0.2 ; \alpha=1 ; c=0.33 ; N_{i}=2$. I choose the triggerprofile $\sigma\left(s^{*}, l^{*}\right)$ that under these conditions is payoff-dominant among all $\sigma(s, l)$ profiles at $\tau=0.95$. This is $s^{*}=6$ and $l^{*}=33$.

\subsubsection{Effects of shadow of the future $(\tau)$}

The feasibility of conditional cooperation depends crucially upon sufficient interest of actors in future outcomes, $\tau$. In the following, it will be analyzed how changes in $\tau$ affect the individual rationality and the payoffs core-members obtain for the profile $\sigma\left(s^{*}, l^{*}\right)$ and for $\sigma_{c o}$. Figure 5.1a shows how changes in $\tau$ affect the payoff-difference $u_{i}-$ $u_{-i}$ for core-members between universal adherence to the trigger strategy and the optimal unilateral deviation for both $\sigma\left(s^{*}, l^{*}\right)$ and $\sigma_{c o}$. Only when this difference is above the zero line, the corresponding profile is an s.p.e. Figure 5.1b shows corresponding changes in the accumulated payoff $u_{i}$ of core-members in the two strategy-profiles.

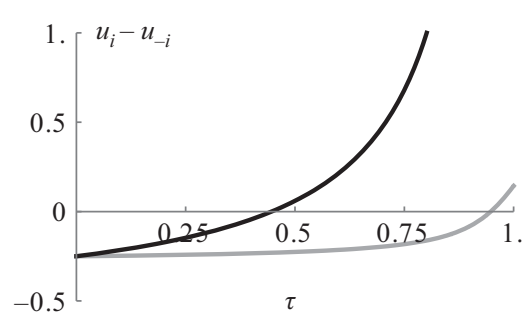

(a)

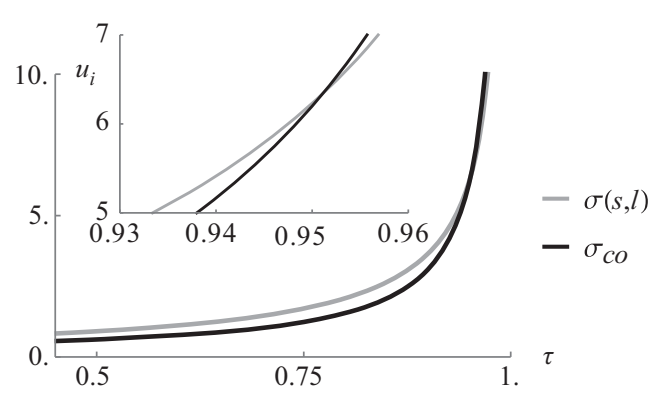

(b)

Figure 5.1: (a): Effect of $\tau$ on payoff difference universal trigger vs. unilateral deviation $u_{i}-u_{-i}$. (b): Effect of $\tau$ on payoff $u_{i}$ for core-members. Inset: range where payoff curves intersect.

For a significant range of $\tau$ (between approximately $\tau=0.45$ and $\tau=0.95$ ) core-only cooperation is found to be payoff inferior to universal cooperation under $\sigma\left(s^{*}, l^{*}\right)$, yet universal cooperation is not individually rational in this range while core-only 
cooperation is. Only when $\tau$ exceeds a critical threshold of about $\tau=0.95$ both profiles are individually rational and universal conditional cooperation becomes the payoff superior solution. However, as $\tau$ further increases, a point is reached where core-only cooperation becomes payoff-superior to universal cooperation (see inset Figure 5.1b). This reflects that if interest in the future is sufficiently strong, the loss of output caused by free riding of two isolates is outweighed by the gain from avoiding frequent punishment periods.

\subsubsection{Effects of number of isolates $\left(N_{i}\right)$ and of noise $(\varepsilon)$ in baseline-scenario}

The baseline scenario suggests that core-only cooperation is the only sustainable solution for a considerable range of conditions and even becomes the most efficient solution when $\tau$ is high enough. The main advantage of the core-only solution is the avoidance of frequent punishment phases caused by uncertainty, while its main disadvantage is the loss of the contributions of peripheral members. Intuitively, a larger share of isolates should then reduce the relative attractiveness of a core-only solution, while more uncertainty should increase it. In the following it will be demonstrated that the game-theoretical model is consistent with these intuitions for the baseline scenario. Figure 5.2 shows how the number of isolates $N_{i}$ affects the individual rationality and efficiency of $\sigma\left(s^{*}, l^{*}\right)$ and of $\sigma_{c o}$ at the level of $\tau=0.95$ and how this effect interacts with a change in the degree of uncertainty from $\varepsilon=0.2$ to $\varepsilon=0.25$. As $\sigma(33,6)$ is s.p.e. throughout in Figure 5.2, the figures show only the accumulated payoff $u_{i}$ for this profile. For $\sigma_{c o}$ both the payoff-difference $u_{i}-u_{-i}$ and the corresponding accumulated payoff $u_{i}$ for core-members are shown. Only when $u_{i}-u_{-i}$ is above zero, $\sigma_{c o}$ is an s.p.e., only when $u_{i}$ for $\sigma_{c o}$ is above $u_{i}$ for $\sigma\left(s^{*}, l^{*}\right)$ in figure 5.2, core-only conditional cooperation is also payoff-superior to universal conditional cooperation.
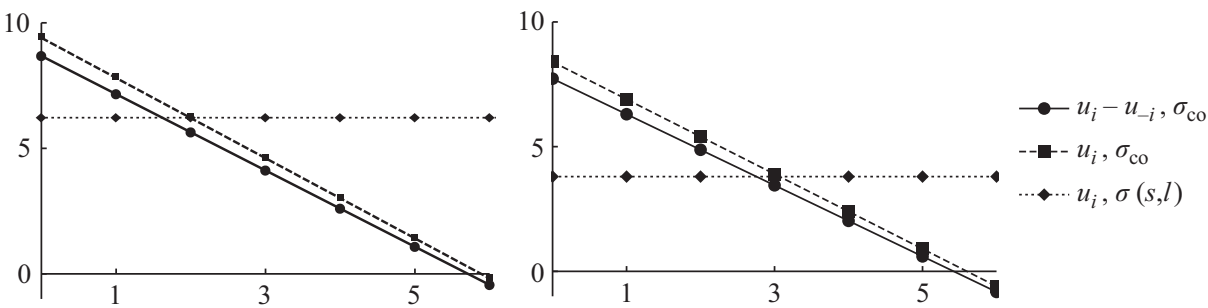

Figure 5.2: Effect of $N_{i}$ on accumulated payoff for both $\sigma\left(s^{*}, l^{*}\right)$ and $\sigma_{c o}$, and effect on $u_{i}-u_{-i}$ for $\sigma_{c o}$. Baseline scenario $N=10 ; \alpha=1 ; c=0.33 ; \tau=0.95, s=33, l=6$. (a) $\varepsilon=0.2$, (b): $\varepsilon=0.25$.

Figure 5.2 demonstrates how core-only conditional cooperation can be both individually rational and payoff-superior to universal conditional cooperation, but only as long as the number of isolates is sufficiently small. For $\varepsilon=0.2, \sigma_{c o}$ is individually 
rational for $N_{i} \leq 5$, and it is payoff-superior to $\sigma\left(s^{*}, l^{*}\right)$ for $N_{i} \leq 1$. If the level of uncertainty increases to $\varepsilon=0.25$, core-members benefit less from cooperation in $\sigma_{c o}$, but also efficiency-losses of $\sigma\left(s^{*}, l^{*}\right)$ become higher. As a consequence, the range within which $\sigma_{c o}$ is the solution of the game widens considerably. The range within which $\sigma_{c o}$ is both individually rational and payoff-superior increases from $N_{i} \leq 1$ for $\varepsilon=0.2$ to $N_{i} \leq 3$ for $\varepsilon=0.25$. Notice that based on condition (5.3) it is straightforward to prove analytically for all feasible parameter vectors that s.p.e. conditions for $\sigma_{c o}$ become more restrictive both for increasing $N_{i}$ as well as for increasing $\varepsilon$. I cannot offer a corresponding analytical result for the payoff-superiority of $\sigma_{c o}$ over $\sigma\left(s^{*}, l^{*}\right)$ but numerical analyses suggest that the results shown here for the baseline scenario generalize to a much wider range of conditions.

\subsubsection{Comparative statics}

\subsubsection{Overview}

In this section comparative statics will be reported about the effects of uncertainty, $\varepsilon$, and the number of isolates, $N_{i}$, on the range of possible values of $\tau$ for which either the optimal $\sigma(s, l)$ profile $\sigma\left(s^{*}, l^{*}\right)$, or $\sigma_{c o}$ or full defection is the solution of the game. The parameters that are fixed are again taken from the baseline scenario, that is $N=10, \alpha=1, c=0.33$. Other than in the analysis of illustrative scenarios, the optimal profile $\sigma\left(s^{*}, l^{*}\right)$ is now computed separately for every point in the parameter space, including for different levels of $\tau$. Both sanctioning time $s$ and cut-off level $l$ can therefore differ across conditions, reflecting the adaptation of the optimal sanctioning profile to the changing requirements for deterrence and lenience as uncertainty and number of isolates change.

The share of the interval $[0,1]$ of possible values of $\tau$ for which a particular type of strategy-profile is the solution of the game will also be used to obtain a coarsegrained indicator of the level of expected group-output $o$ per round at a particular point in the parameter space. More precisely, for every level of $\tau$, the corresponding solution of the game is identified and the related expected group-output $o(\tau)$ per round is computed. Overall expected group-output $o$ at $\left(\alpha, c, N, N_{c}, \varepsilon\right)$ is then computed as average across the entire range of $\tau$. For convenience the expected output is linearly rescaled to $[0,1]$. Details of the method how $o(\tau)$ is computed for a given profile $\sigma(s, l)$ can be found in Flache (2002). When the solution is the optimal $\sigma(s, l)$ profile, expected output is obtained as the probability that a particular round of the game falls within a normal period, multiplied with the rescaled expected groupoutput $(1-\varepsilon)$ in a normal round. Finally, if the solution is $\sigma_{c o}$, expected output is taken as $(1-\varepsilon) N_{c} / N$.

To distinguish between output based on universal conditional cooperation and output based on core-only conditional cooperation, I also adapt the method described 
above to obtain two additional output indicators. These indicate expected outputs separately computed for the fraction of the range of $\tau$ in which $\sigma(s, l)$ is s.p.e., and in which $\sigma_{c o}$ is s.p.e. These indicators are denoted $o_{\sigma}$ and $o_{c o}$, respectively.

\subsubsection{Comparative statics for the effects of uncertainty $\varepsilon$}

Figure 5.3 shows how the output indicators as well as the distribution of solutions in the $\tau$-space change when uncertainty increases from $\varepsilon=0$ to $\varepsilon=0.5$, given $N=10$, $\alpha=1, c=0.33$ and $N_{i}=2$. More precisely, for this analysis both $\varepsilon$ and $\tau$ are varied across 100 equidistant steps.

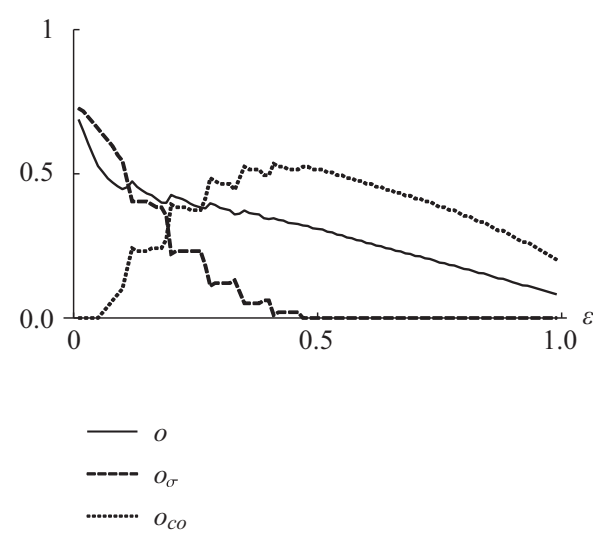

(a) expected output
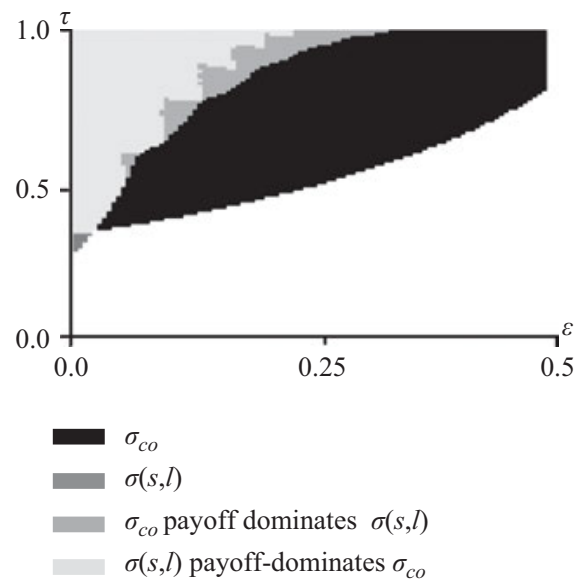

(b) solutions in $\varepsilon-\tau$ space

Figure 5.3: Effect of uncertainty $\varepsilon$ on indicators of expected output and distribution of solutions in $\varepsilon-\tau$ parameter space. Baseline scenario: $N=10 ; \alpha=1 ; c=0.33 ; N_{i}=2$.

Figure 5.3b shows that at all levels of $\varepsilon$, any form of conditional cooperation is sustainable only when $\tau$ exceeds a critical threshold that becomes increasingly restrictive as $\varepsilon$ increases. The figure also shows that except for very low levels of uncertainty, the conditions under which $\sigma_{c o}$ is the solution of the game are less restrictive than are the conditions for $\sigma(s, l)$. For approximately $0.02<\varepsilon<0.3, \sigma(s, l)$ is s.p.e. in an intermediate range of $\tau$, but in parts of this region it is still payoff-dominated by $\sigma_{c o}$. The erratic shape of those areas in Figure 5.3b is due to the discrete structure of the strategy space for $\sigma(s, l)$. For the uncertainty range of $0.02<\varepsilon<0.3$, the profile $\sigma(s, l)$ becomes the payoff-dominant solution only when $\tau$ exceeds an even higher threshold. From about $\varepsilon=0.3$ on, $\sigma(s, l)$ can no longer be sustained for any level of $\tau$, while this is still possible for $\sigma_{c o}$. These results confirm the intuition that universal conditional cooperation is 
less robust against uncertainty than core-only conditional cooperation. Figure 5.3a charts how this affects expected outputs $o, o_{\sigma}$ and $o_{c o}$. As uncertainty increases, expected output drops both overall and for $o_{\sigma}$. The output indicator $o_{c o}$ shows a different pattern. It first increases, then decreases in uncertainty. The reason is that starting from zero uncertainty, more uncertainty quickly widens the range of conditions under which core-only cooperation is the only individually rational strategy profile. Only beyond approximately $\varepsilon=0.2$, efficiency losses from uncertainty also noticeably affect the expected output $o_{c o}$ that is sustained by the core-only profile.

\subsubsection{Comparative statics for the effects of the number of isolates $\mathbf{N}_{i}$}

Figure 5.4 shows how changes in the number of isolates between $N_{i}=0$ and $N_{i}=8$ affect the distribution of solutions and expected output for the baseline-scenario $N=10, \alpha=1, c=0.33$ and $\varepsilon=0.2$. Both $N_{i}$ and $\tau$ are varied across 100 equidistant steps in this analysis. While the number of isolates is conceptually a variable with only integer values, mathematically the corresponding indicators can also be computed for non-integer $N_{i}$. Quasi-continuous $N_{i}$ was therefore used here to obtain a smooth representation of results.

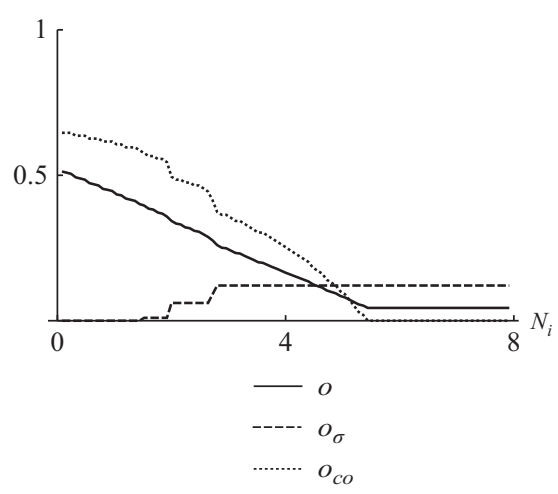

(a) expected output

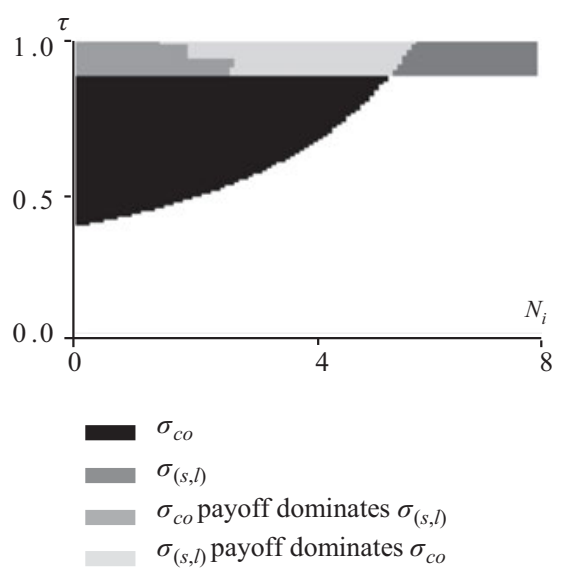

(b) solutions in $N_{i}-\tau$ space

Figure 5.4: Effect of number of isolates $N_{i}$ on indicators of expected output and distribution of solutions in $N_{i}-\tau$ parameter space. Baseline scenario: $N=10 ; \alpha=1 ; c=0.33 ; \varepsilon=0.2$.

Figure 5.4b shows that the range of conditions under which universal conditional cooperation $\sigma(s, l)$ yields an individually rational strategy-profile is not affected by $N_{i}$. In the baseline-scenario, $\tau$ needs to exceed approximately $\tau=0.86$ to sustain 
cooperation based on $\sigma(s, l)$, regardless of the value of $N_{i}$. The number of isolates has no effect on the rationality condition for $\sigma(s, l)$. In this profile, group members condition sanctioning only on the observed group-output. This implies that the sanctioning profile needed to keep a group member in line is the same for both core-members and isolates. Moreover, as long as the group contains at least one isolate there is also no other symmetric as well as efficient profile that guarantees that all group members including the isolate cooperate conditionally. Figure $5.4 \mathrm{~b}$ demonstrates furthermore that the conditions under which $\sigma_{c o}$ is individually rational are less restrictive than those for $\sigma(s, l)$ as long as $N_{i} \leq 5$, but unlike for $\sigma(s, l)$ this range shrinks if a group contains more isolates. This happens because more freeriding isolates reduce the long-term benefits core-members can obtain under $\sigma_{c o}$ relative to the short-term gains from unilateral defection. Beyond $N_{i}=6$, core-only conditional cooperation becomes unsustainable at all levels of $\tau$. The figure further shows a region of conditions under which universal conditional cooperation payoff-dominates core-only cooperation, while both are individually rational. This happens when $N_{i}$ is in an intermediate range (between about 2 and 6) and $\tau$ exceeds approximately $\tau=0.86$. Above this level of $\tau$ it can be observed that the payoffdominance relation between the two profiles is reversed for lower levels of $N_{i}$, because here efficiency losses under $\sigma_{c o}$ are relatively small compared to efficiency losses from sanctioning periods under $\sigma(s, l)$. At higher levels of $N_{i}, \sigma_{c o}$ is no longer sustainable and $\sigma(s, l)$ becomes the unique conditionally cooperative profile that is individually rational.

Figure 5.4a shows how the negative impact of $N_{i}$ on core-only cooperation translates into a negative impact on the expected output a group with a core-periphery structure can achieve. While larger $N_{i}$ comes with higher $o_{\sigma}$ from about $N_{i}=1.5$ on, the contribution that universal conditional cooperation can make to group-output is too small to compensate for the output-losses that core-only cooperation suffers from an increasing number of free-riding isolates.

All in all, Figure 5.4 confirms the intuition that a larger number of isolates makes core-only cooperation harder to attain and less efficient, such that universal conditional cooperation can become the superior solution given that group members are sufficiently interested in future outcomes.

\subsection{Discussion and conclusion}

Conditional cooperation can be a powerful endogenous solution to the problem of collective action if interactions within a group are "temporally embedded" (Batenburg et al. 2003). However, as several authors have pointed out, the level of provision of a collective good that can be sustained by endogenous conditional cooperation may be severely reduced if uncertainty blurs the link between the observed provision level of 
a collective good and the underlying individual contribution decisions. Moreover, such uncertainty may also considerably reduce the range of conditions under which endogenous conditional cooperation is feasible at all for individually rational actors. In this case embeddedness in social networks can be an important condition that stabilizes conditional cooperation. Following up on Raub \& Weesie's (1990) analysis, a large number of theoretical and empirical papers demonstrated that monitoring and control via social network relations can safeguard conditional cooperation especially for two-party cooperation problems, even when information about the degree of cooperation at the collective level is unreliable or not available.

In this paper I have proposed a first step towards integrating monitoring via direct social ties into a model of conditional cooperation in collective-good production under uncertainty. The model proposed here points to a dilemma that may occur especially in groups with a core-periphery structure. Members embedded in the densely connected core of a wider network face closer monitoring and can be more effectively sanctioned under conditional cooperation than members in peripheral positions in the network. To keep the latter in line, harsher sanctioning regimes must be imposed but these entail under uncertainty the possible cost of frequent 'erroneous sanctioning' which is detrimental for all group members. Thus, even for core-members themselves it can be a more attractive rational solution to adopt a cooperation norm that enforces contributions from core-members only and tolerates free-riding from peripheral members of the group. Yet, such an outcome can be deemed unfair, which imposes another social cost on the group.

The analysis presented here may point to testable hypotheses for empirical studies that aim to explain differences in cooperation in collective action between otherwise comparable groups that are different in their network structures. One example are organizations, like university departments, which can differ from each other in the relative number of full-time and part-time employees. Another are voluntary community-based initiatives, like local renewable energy initiatives, some of which operate in cohesive rural communities and some in more sparsely connected urban neighborhoods. A complication in formulating such hypotheses is that in a concrete collective good problem there are many other characteristics of actors that may affect contribution to a collective effort and are correlated the same time with actors' position in a network. For example, members of a team of frontrunners in a community-energy initiative are likely to attach more value to the collective good of protection of the environment than community members who do not belong to this core-team, which by itself may explain why they would be more willing to contribute. However, the model proposed here also points to potentially unique testable implications. For example, it suggests that front-runners in a community energy initiative might be more willing to accept low levels of contributions from other community members without trying to respond with sanctions, the larger the core-team is relative to the community as a whole and the less easy it is for members of the core team to observe the reasons why others fail to contribute. At the 
same time, their own level of contribution should not be reduced by those conditions. However, before empirical applications can be seriously addressed, a number of strong simplifications and potential limitations of the analysis presented here require careful inspection.

A first possible limitation is that I have focused only on a limited set of possible conditionally cooperative strategies. Obviously other and more complex conditionally cooperative strategy-profiles can be constructed. Especially profiles are of interest that combine the sanctioning-threat that core-only cooperation imposes on core-members with more lenient yet demanding norms for contributions by isolates. Such profiles could be more efficient than $\sigma_{c o}$ as well as less vulnerable to uncertainty than $\sigma(s, l)$. However, while this possibility cannot be excluded it should also be pointed out that such profiles can lead to prohibitively complex coordination problems as they involve different $s$ and $l$ levels for the different types of actors, as well as different degrees of contribution and sanctioning from core-members and isolates. This higher coordination complexity reduces the empirical plausibility of such solutions.

A second strong simplification I have adopted is the maximally simple network structure that distinguishes only two types of network positions, core-members and isolates. In real groups facing collective good problems networks are more heterogeneous. The analysis proposed here could be extended to such settings with the assumption that core-members will always defect if they observe in their personal network at least one verifiable defection. In this case, a defection of a core-member would trigger off a 'wave' of defection spreading through the network. Intuitively, this implies that members who are more closely connected also face stronger incentives to cooperate conditionally, because their defection leads to a faster and possibly more comprehensive breakdown of cooperation than the defection of more peripheral or even isolated network-members. On a qualitative level this retains the prediction of my simpler model that conditions for universal conditional cooperation are more restrictive in less densely connected networks, similar to results obtained in the research on effects of network embeddedness on cooperation in twoparty interactions.

A third simplification is that my model abstracts from other differences between group members than their position in the network. For many settings it is plausible that that the network position is correlated with other differences between group members. One example is an organization in which members with longer tenure both face a darker shadow of the future and hold more central network positions than temporary workers. Another is a voluntary association, in which members with a larger interest in the collective good are also more active and therefore form part of the core of a network of activists. Moreover, mutual sanctioning and control among core-members can be expected to be even more effective if it is taken into account that network ties serve not only as channels for monitoring but also for imposing direct positive or negative sanctions upon other network-members (Coleman 
1990; Fehr and Gächter 2002; Flache 1996; Flache et al. 2017). As long as more central network positions come with stronger incentives to contribute to the collective good (as in these examples), additional heterogeneity would not alter the proposition of a rational exploitation of the core by the periphery. However, more central network positions may also help core-members to enforce peripheral members' contributions more effectively. One possibility is that core-members hold positions with more formal sanctioning power in an organization, another that they use their social ties to coordinate effective monitoring and sanctioning efforts directed towards peripheral members (Coleman 1990). In addition, especially in organizations peripheral workers with temporary or part-time contracts may have strong incentives to contribute to collective organizational goods because this can enhance their prospect of acquiring tenure or other future rewards from the employer (cf. Lambooij, Flache, and Siegers 2009).

Fourth, a strong simplification of the model developed here is that networks are assumed to be static. In several of the examples discussed above, it would be possible that members of a group strategically change their network ties, for example in order to increase their possibility to observe others' contributions. Or, a principal could change organizational structures to reduce the isolation of peripheral members aiming to thereby facilitate endogenous cooperation. The very structures that inhibit conditional cooperation according to the model proposed here, could also be the outcome of processes of endogenous strategic network formation in which cooperators try to evade interactions with defectors (Sohn, Choi and Ahn 2019), or in which defectors try to insulate themselves from outside pressures to contribute (Takács, Janky and Flache 2008).

A last complication worth considering is the possibility of ostracizing isolates who free-ride (Hirshleifer and Rasmusen 1989). After all, under a core-only norm these members add nothing to the group-output. While this may be a readily available solution in a group that focuses only on one collective good to produce, in many empirical situations peripheral group members may make other valuable contributions that are unrelated to a specific collective good. Think for example of a university department that desperately needs temporarily employed teachers to offer all the courses needed, but also would like all employees to contribute to more-or-less voluntary collective activities such as organizing departmental colloquia or attending staff-meetings. It is quite plausible that in such cases norms emerge that demand perpetual contribution from tenured staff but tolerate some level of free-riding from less well connected temporary members of the organization.

Despite the limitations of the simple model I have proposed here, I hope to have demonstrated that studying how networks may sustain conditional cooperation through improved possibilities for monitoring is potentially a fruitful source of inspiration not only for the area of trust in two-party relations, but also for the study of cooperation in the production of collective goods. 


\section{References}

Axelrod, Robert. 1984. The Evolution of Cooperation. New York: Basic Books.

Batenburg, Ronald S., Werner Raub and Chris Snijders. 2003. "Contacts and Contracts: Dyadic Embeddedness and the Contractual Behavior of Firms." Research in the Sociology of Organizations 20: 135-88.

Bednar, Jenna. 2006. "Is Full Compliance Possible?: Conditions for Shirking with Imperfect Monitoring and Continuous Action Spaces." Journal of Theoretical Politics 18 (3): 347-75.

Bendor, Jonathan and Dilip Mookherjee. 1987. "Institutional Structure and the Logic of Ongoing Collective Action." American Political Science Review 81 (1): 129-54.

Bendor, Jonathan and Dilip Mookherjee. 1990. "Norms, Third-Party Sanctions, and Cooperation." Journal of Law, Economics, and Organization 6 (1): 33-63.

Bendor, Jonathan, Roderick M. Kramer and Suzanne Stout. 1991. "When in Doubt . . . : Cooperation in a Noisy Prisoner's Dilemma." Journal of Conflict Resolution 35 (4): 691-720.

Buskens, Vincent. 2002. Social Networks and Trust. Dordrecht: Kluwer Academic Publishers.

Buskens, Vincent and Werner Raub. 2002. "Embedded Trust: Control and Learning." Pp. 167-202. In Group Cohesion, Trust and Solidarity. Advances in Group Processes, ed. Shane R. Thye and Edward J. Lawler. Amsterdam: Elsevier.

Chwe, Michael S-Y. 1999. "Structure and Strategy in Collective Action." American Journal of Sociology 105 (1): 128-56.

Coleman, James S. 1990. Foundations of Social Theory. Cambridge, Ma.: Harvard University Press.

Dijkstra, Jacob and Marcel A.L.M. van Assen. 2013. "Network public goods with asymmetric information about cooperation preferences and network degree." Social Networks 35 (4): 573-582.

Fatas, Enrique, Miguel A. Meléndez-Jiménez, and Hector Solaz. 2010. "An experimental analysis of team production in Networks." Experimental Economics 13 (4): 399-411.

Fatas, Enrique, Miguel A. Meléndez-Jiménez, Antonio Morales and Hector Solaz. 2015. "Public Goods and Decay in Networks." SERIEs: Journal of the Spanish Economic Association 6 (1): 73-90.

Fehr, Ernst and Simon Gächter. 2002. “Altruistic Punishment in Humans.” Nature 415 (6868): 137-40.

Flache, Andreas. 1996. The Double Edge of Networks. An Analysis of the Effect of Informal Networks on Cooperation in Social Dilemmas. Amsterdam: Thesis Publishers.

Flache, Andreas. 2002. "The Rational Weakness of Strong Ties: Collective Action Failure in a Highly Cohesive Group of Rational Agents." Journal of Mathematical Sociology 26: 189-216.

Flache, Andreas. 2004. "How May Virtual Communication Shape Cooperation in a Work Team? A Formal Model Based on Social Exchange Theory." Analyse \& Kritik 26 (1): 258-78.

Flache, Andreas, Dieko Bakker, Michael Mäs and Jacob Dijkstra. 2017. "The Double Edge of Counter-Sanctions. Is Peer Sanctioning Robust to Counter-Punishment but Vulnerable to Counter-Reward?" Pp. 280-301 in Social Dilemmas, Institutions, and the Evolution of Cooperation, ed. by Ben Jann and Wojtek Przepiorka. Berlin: De Gruyter Oldenbourg.

Flache, Andreas, Michael W. Macy and Werner Raub. 2000. "Do Company Towns Solve Free Rider Problems? A Sensitivity Analysis of a Rational-Choice Explanation." Pp. 123-125 (summary accompanied by full paper on CD-ROM, 36 pp.). In: The Management of Durable Relations: Theoretical and Empirical Models for Households and Organisations, ed. Jeroen Weesie and Werner Raub. Amsterdam: Thela Thesis.

Friedman, James W. 1971. "A Non-Cooperative Equilibrium for Supergames." Review of Economic Studies 38: 1-12. 
Friedman, James W. 1986. Game Theory with Applications to Economics. New York: Oxford University Press.

Goedkoop, Fleur, Andreas Flache, and Jacob Dijkstra. 2017. "Participation within Community led Energy Projects: The Role of Social Networks. Paper presented at Third European Conference on Social Networks - EUSN 2017. Mainz, September 28, 2017.

Gould, Roger V. 1993. “Collective Action and Network Structure.” American Sociological Review 58: 182-96.

Granovetter, M. 1985. "Economic Action and Social Structure : The Problem of Embeddedeness." American Journal of Sociology 91: 481-512.

Green, Edward J., and Robert H. Porter. 1984. "Noncooperative Collusion under Imperfect Price Information." Econometrica 52 (1): 87-100.

Hardin, Garrett. 1968. "The Tragedy of the Commons." Science 162: 1243-48.

Harsanyi, John C. 1977. Rational Behavior and Bargaining Equilibrium in Games and Social Situations. Cambridge: Cambridge University Press.

Hirshleifer, David, and Eric Rasmusen. 1989. “Cooperation in a Repeated Prisoners' Dilemma with Ostracism.” Journal of Economic Behavior and Organization 12: 87-106.

Kollock, Peter. 1993. “'An Eye for an Eye Leaves Everyone Blind' : Cooperation and Accounting Systems." American Sociological Review 28: 768-86.

Kreps, David M. 1990. A Course in Microeconomic Theory. New York: Harvester.

Lambooij, Mattijs, Andreas Flache and Jacques Siegers. 2009. "Shadow of the Future, Risk Aversion, and Employee Cooperation." Rationality and Society 21 (3): 307-336.

Macy, Michael W. 1991. "Chains of Cooperation : Threshold Effects in Collective Action." American Sociological Review 56: 730-47.

Marwell, Gerald, and Pamela Oliver. 1993. The Critical Mass in Collective Action. A Micro-Social Theory. Cambridge (Mass.): Cambridge University Press.

Nikiforakis, Nikos. 2008. "Punishment and Counter-Punishment in Public Good Games: Can We Really Govern Ourselves?” Journal of Public Economics 92 (1): 91-112.

Nikiforakis, Nikos and Dirk Engelmann. 2011. "Altruistic Punishment and the Threat of Feuds." Journal of Economic Behavior and Organization 78 (3): 319-32.

Olson, M. 1965. The Logic of Collective Action. Cambridge, MA: Harvard University Press.

Raub, Werner. 1988. "Problematic Social Situations and the Large Number Dilemma." Journal of Mathematical Sociology 13 (4): 311-57.

Raub, Werner. 2017. Rational Models. Utrecht: Universiteit Utrecht.

Raub, Werner and Thomas Voss. 1986. "Conditions for Cooperation in Problematic Social Situations." In Paradoxal Effects of Social Behavior. Essays in Honor of Anatol Rapoport, edited by A Rapoport, Andreas Diekmann, and P Mitter, 85-103. Heidelberg: Physica-Verlag.

Raub, Werner and Jeroen Weesie. 1990. "Reputation and Efficiency in Social Interactions : An Example of Network Effects." American Journal of Sociology 96 (3): 626-54.

Raub, Werner and Jeroen Weesie. 2000. "The Management of Matches: A Research Program on Solidarity in Durable Social Relations." Netherlands' Journal of Social Sciences : A Publication of the Netherlands' Sociological and Anthropological Society 36 (1): 71-88.

Raub, Werner, and Vincent Buskens. 2008. "Theory and Empirical Research in Analytical Sociology: The Case of Cooperation in Problematic Social Situations." Analyse \& Kritik 30 (2): 689-722.

Rooks, Gerrit, Werner Raub and Frits Tazelaar. 2006. "Ex Post Problems in Buyer-Supplier Transactions: Effects of Transaction Characteristics, Social Embeddedness, and Contractual Governance." Journal of Management and Governance 10: 239-76.

Selten, Reinhard. 1965. "Spieltheoretische Behandlung Eines Oligopolmodells Mit Nachfrageträgheit." Zeitschrift Für Die Gesamte Staatswissenschaft 121: 301-24. 
Sohn, Yunkyu, Jung-Kyoo Choi and Toh-Kyeong Ahn. 2019. "Core-Periphery Segregation in Evolving Prisoner's Dilemma Networks". Journal of Complex Networks 8 (1): cnz021, https://doi.org/10.1093/comnet/cnz021.

Spagnolo, G. 1999. "Social Relations and Cooperation in Organizations." Journal of Economic Behavior \& Organization 38: 1-25.

Takács, Karoly, Béla Janky and Andreas Flache. 2008. "Collective Action and Network Change." Social Networks 30 (3):177-189.

Taylor, Michael. 1976. Anarchy and Cooperation. London UK: Wiley \& Sons.

Taylor, Michael. 1987. The Possibility of Cooperation. Cambridge: Cambridge University Press.

Wu, Jianzhong and Robert Axelrod. 1995. "How to Cope with Noise in the Iterated Prisoner's Dilemma. Journal of Conflict Resolution 39 (1): 183-189.

Wolitzky, Alexander. 2013. “Cooperation with Network Monitoring." Review of Economic Studies 80 (1): 395-427. 